ACTA AGROBOTANICA

Vol. 62 (1): 171-186

2009

\title{
SEGETAL COMMUNITIES OF CEREAL CROPS OF THE MAZOWIECKI LANDSCAPE PARK
}

\author{
Teresa Skrajna, Janina Skrzyczyńska, Maria Ługowska
}

The Agricultural Ecology Department of the University of Podlasie in Siedlce

B. Prusa 14, 08-110 Siedlce, Poland, e-mail: ekorol@ ap.siedlce.pl

Received: 11.07.2008

Abstract

The characteristics of communities developing in cereal crops of the Mazowiecki Landscape Park are presented in the paper. The traditional methods of cultivation, a mosaic of fields, meadows and forests as well as a specific microclimate affect the occurrence of species-rich, syntaxonomically differentiated agrophytocenoses. The occurrence of 4 associations and 2 communities in the area of the Park was noted. On the poorest soils, the association Arnoserido-Scleranthetum was the most widespread and internally differentiated, whereas in more fertile habitats the association Vicietum tetraspermae was the commonest. The above mentioned associations were noted more frequently in winter crops than in spring cereals. Plots of the association Papaveretum argemones and intermediate communities, between Arnoserido-Scleranthetum and Papaveretum argemones, were seldom observed in the studied area. Plots with domination of species diagnostic for the order Polygono-Chenopodietalia occurred rarely in spring cereals, mainly in the protection zone of the Landscape Park. Single, impoverished plots of Aphano-Matricarietum were recorded in the south-eastern part of the Park.

Key words: segetal vegetation, cereal associations, intermediate communities, Mazowiecki Landscape Park

\section{INTRODUCTION}

The Mazowiecki Landscape Park was established in 1986. The Park is situated in the central-eastern part of Mazowsze Province. It covers an area of 15710 ha.

Arable lands occupy only a little part of the Mazowiecki Landscape Park (20\% of its area). They are situated mainly in the environs of Polana Ponurzycka (in the southern part of the Park), in the vicinity of Aleksandrów (in its northern part), as well as in its agricultural buffer zone. The acreage of arable lands constantly decreases, which is the result of cultivation abandonment and intensive residential building development.
Arable lands are differentiated as to their agricultural value and farming intensity. Soils of rye complexes: very poor, poor and good, as well as a poor cereal-grazing complex prevail in the study area. Small fragments of agriculturally favourable, more productive soils of very good rye and good wheat complexes were rarely observed, especially in the south-eastern part of the Park, in the environs of Regut village. Peatbogs and boggy soils occurring between the localities Brzezinka and Całowanie in the west and Tabor and Podbiel in the east of the Park were also noted. Small and medium-sized farms with traditional methods of cultivation dominate in the area of the Landscape Park. The occurrence of well developed, floristically differentiated communities in cereal agrocenoses is the result of extensive farming methods.

The aim of the work is phytosociological classification of patches of vegetation occurring in cereal agrocenoses as well as the characteristics of internal differentiation of syntaxa against a background of habitat conditions.

\section{METHODS}

Field studies were carried out between 2003 and 2007 in agrocenoses of the Mazowiecki Landscape Park and its buffer zone. In total, in cereal crops of the studied area 119 phytosociological relevés were made according to the Braun-Blanquet method (Pawłowski, 1972). Soil type was determined on the basis of soil-agricultural maps at a scale of 1:5000. Collected documentation material was systematized according to the Matuszkiewicz classification (2001). Species nomenclature followed M i r e k et al. (2005). The distribution of the studied localities is presented at Fig. 1. 


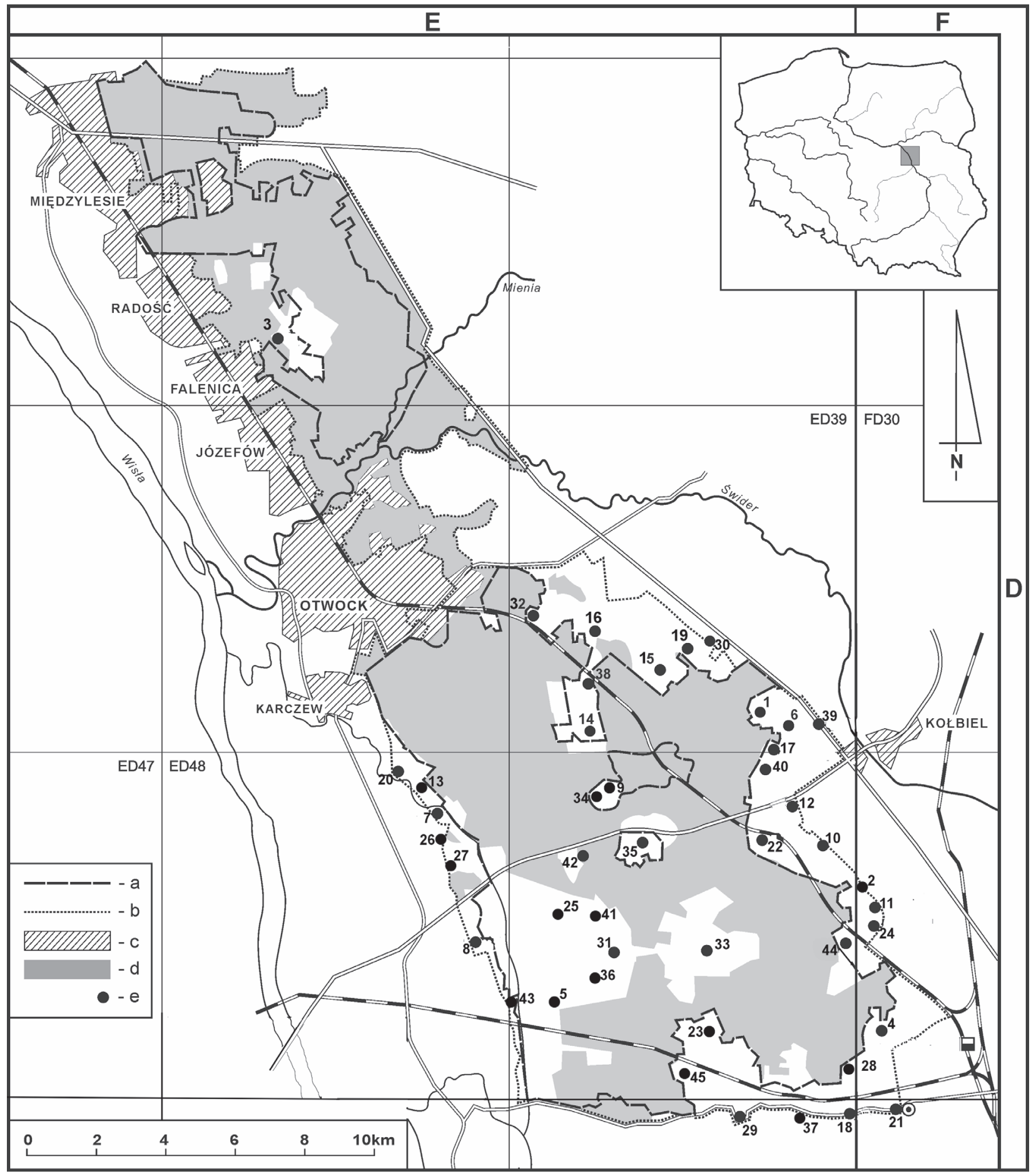

Fig. 1. Study area

$\mathrm{a}$ - borders of the Landscape Park; $\mathrm{b}$ - borders of the protected zone; $\mathrm{c}$ - building land;

$\mathrm{d}$ - forest complexes; e - localities, investigated areas.

1 - Anielinek, 2 - Antoninek, 3 - Aleksandrówka, 4 - Augustówka, 5 - Bąki, 6 - Bocian, 7 - Brzezinka, 8 - Całowanie, 9 - Celestynów, 10 - Chrosna, 11 - Chrząszczówka, 12 - Człekówka, 13 - Dąbrowa, 14 - Dąbrówka, 15 - Dyzin, 16 - Glina, 17 - Gózd, 18 - Grabianka, 19 - Jatne, 20 Janów, 21 - Jaźwiny, 22 - Karpiska, 23 - Kąciki, 24 - Kąty, 25 - Kominki, 26 - Kozłówka, 27 - Łukowiec, 28 - Ocznia, 29 - Osieck, 30 - Ostrowik, 31 - Podbiel, 32 - Pogorzel, 33 - Ponurzyca, 34 - Radzyń, 35 - Regut, 36 - Rosłańce, 37 - Rudnik, 38 - Stara Wieś, 39 - Stara Wieś II, 40 - Skorupy, 41 - Szatany, 42 - Tabor, 43 - Warszówka, 44 - Zabieżki, 45 - Zawada. 


\section{RESULTS}

Systematic of the distinguished cereal associations and communities

Class: Stellarietea mediae Tx., Lohm. et Prst. 1950

Order: Centauretalia cyani R. Tx. 1950

Alliance: Aperion spicae-venti R. Tx. et. J. Tx. 1960

1. Association: Arnoserido-Scleranthetum (Edouard 1925) R. Tx. 1937

a. typical variant

b. variant with Polygonum hydropiper

c. variant with Anthoxanthum aristatum

Intermediate community Arnoserido-Scleranthetum

(Edouard 1925) R. Tx. 1937 -

Papaveretum argemones (Libb. 1932)

Krusem. et Vlieg. 1939

2. Association: Papaveretum argemones (Libb. 1932) Krusem. et Vlieg. 1939

3. Association: Vicietum tetraspermae (Krusem. et Vlieg. 1939) Kornaś 1950

a. typical variant

b. variant with Juncus bufonius

c. variant with Anthoxanthum aristatum

d. variant with Rhinanthus serotinus

- subvariant with Myosurus minimus

4. Association: Aphano-Matricarietum R. Tx. 1937

Community with species characteristic for Polygono-Chenopodion

\section{Characteristics of the distinguished associations and communities}

Arnoserido-Scleranthetum (Edouard 1925) R. Tx. 1937

Patches of Arnoserido-Scleranthetum were observed commonly in agrocenoses of the Landscape Park. They developed on the poorest soils, formed from non-cohesive and poor-clayey sands classified as very poor and poor rye complexes and poor cereal-grazing complex, noted in thinned out rye crops on mid-forest dunes. These phytocenoses were described based on 30 phytosociological relevés (Tab. 1 ). The differentiation of habitats, in which the association was noted, affects the division of ArnoseridoScleranthetum into 3 variants: with Anthoxanthum aristatum, typical and with Polygonum hydropiper. Arnoserido-Scleranthetum was noted on the borders of the Park and within its protection zone. The floristically-poorest phytocenoses were dominated by $A n-$ thoxanthum aristatum (mean cover in the plot was about $40 \%$ ). In the typical variant, species characteristic for the association Arnoseris minima and Teesdalea nudicaulis reached the largest cover. Patches of the variant with Polygonum hydropiper developed on silt-peat and boggy soils situated in local depressions. Higrophilous species, such as Bidens tripartita, Jun- cus bufonius, Equisetum sylvaticum and others, were the frequent components of this variant. That was the most species-rich phytocenosis of the ArnoseridoScleranthetum associations, made up of 64 species, on average 24 species in one plot. Species such as Apera spica-venti, Centaurea cyanus and Anthemis arvensis occurred most frequently and with the largest cover.

Arnoserido-Scleranhetum (Eduard 1925)R.Tx 1937 - Papaveretum argemones (Libb. 1932) Kruzem. Et Vlieg. 1939

Small patches of phytocenoses of intermediate character, classified in the alliance Aperion spicaeventi, were noted rarely. They developed in habitats characteristic for typical patches of Arnoserido-Scleranthetum and acidophilous patches of Papaveretum argemones (Tab. 2). These phytocenoses were distinguishable by a large share of species characteristic for both the association Arnoserido-Scleranthetum (Arnoseris minima, Teesdalea nudicaulis) and Papaveretum argemones (Arabidopsis thaliana, Veronica triphyllos and Papaver argemone).

Papaveretum argemones (Libb. 1932) Krusem. et Vlieg. 1939

Patches of the the Papaveretum argemones association were noted mainly at Polana Ponurzycka and rarely in the buffer zone of the Park, in the vicinity of Osieck and Stara Wieś (Tab. 3). The phytocenoses of Papaveretum argemones were found in rye crops, in well insolated areas, at local elevations. They developed mainly on brown lixiviated soils composed of gravels and light loamy sands classified as poor and good rye complexes. The phytocenoses were distinguishable by large phenological variability, e.g. the numerous occurrence of characteristic species: Arabidopsis thaliana and Veronica hederifolia in spring, and Papaver argemone - in summer.

Vicietum tetraspermae (Krusem. et Vlieg. 1939) Kornaś 1950

In cereal crops of the Mazowiecki Landscape Park and its buffer zone, phytocenoses of Vicietum tetraspermae were noted frequently. From among the diagnostic species of the association, Vicia tetrasperma was noted most frequently, less frequently - Polygonum lapathifolium subsp. pallidum, sporadically - Bromus secalinus. A large affiliation of distinguishing species - Vicia villosa to the Vicietum tetraspermae association was also observed. A various combination of accompanying species is the reason for the internal division of the association (Tabs 4, 5). 


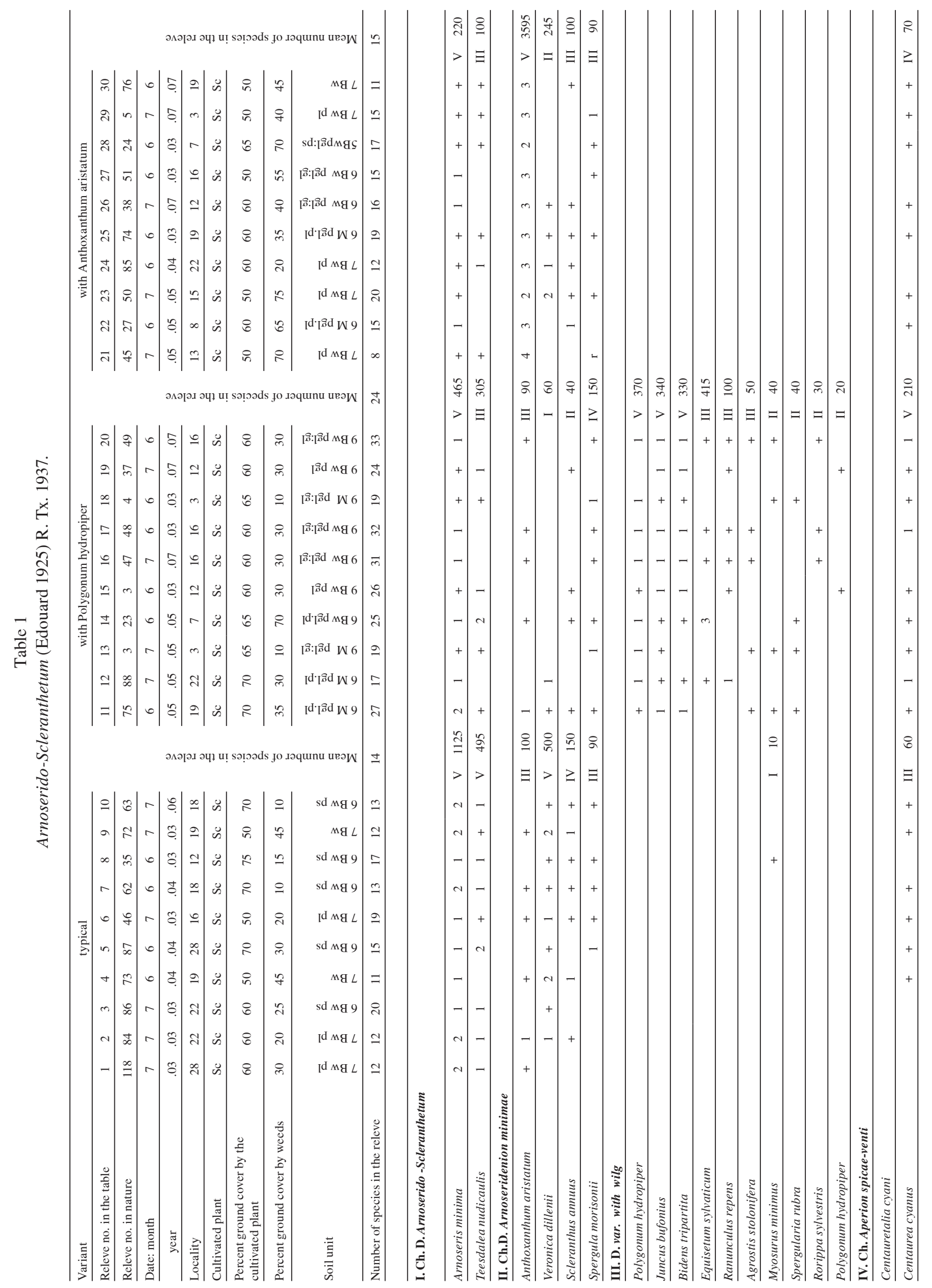




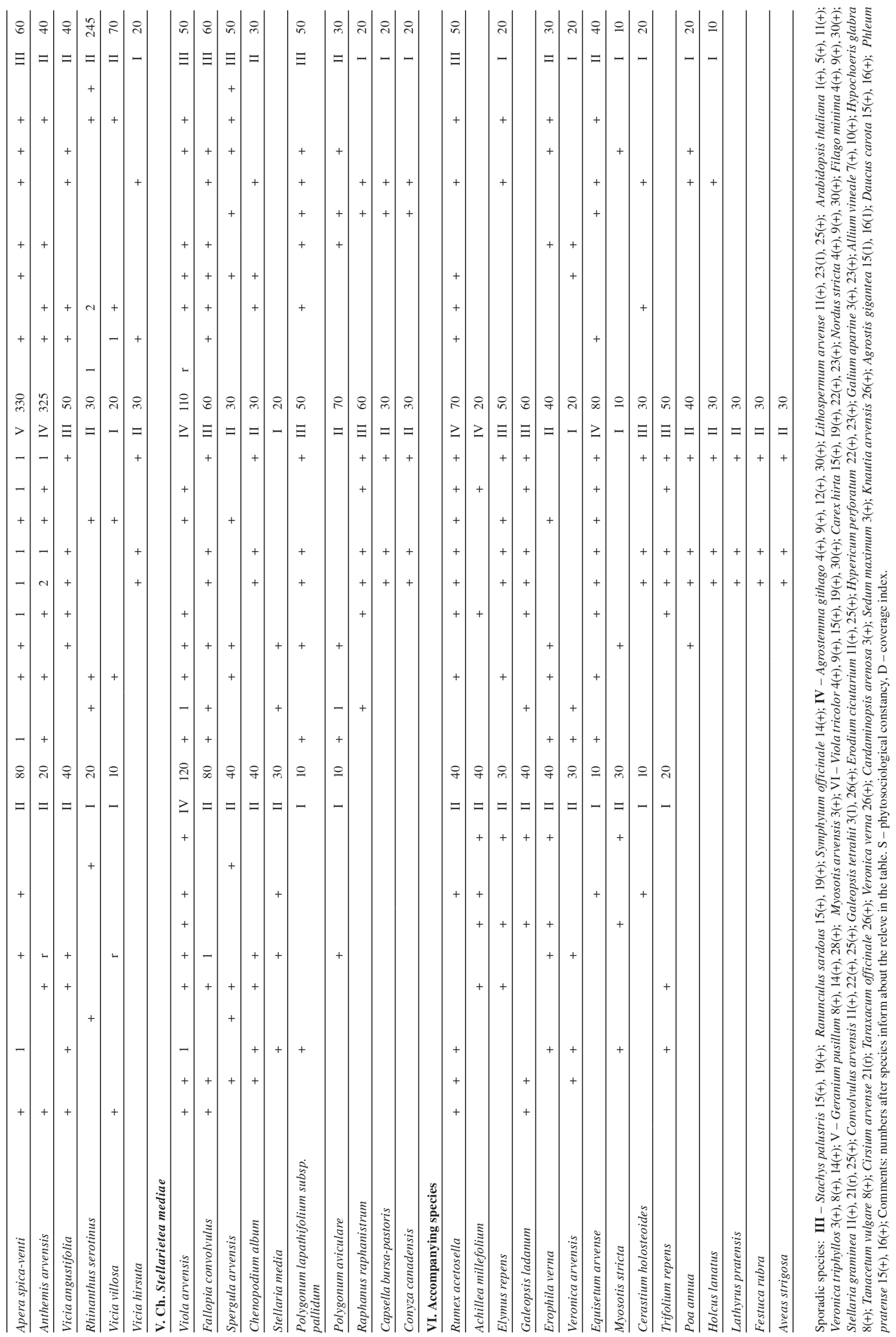


Table 2

Community Arnoserido-Scleranthetum (Edouard 1925) R. Tx 1937 - Papaveretum argemones (Libb. 1932) Kruzem. Et Vlieg. 1939.

\begin{tabular}{|c|c|c|c|c|c|c|c|c|c|c|c|c|}
\hline Releve no. in the table & 1 & 2 & 3 & 4 & 5 & 6 & 7 & 8 & 9 & 10 & \multirow{9}{*}{\multicolumn{2}{|c|}{ 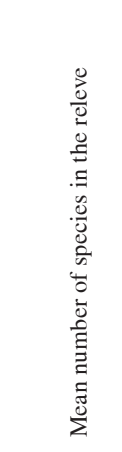 }} \\
\hline Releve no. in nature & 57 & 58 & 25 & 52 & 111 & 26 & 112 & 43 & 99 & 44 & & \\
\hline Date: month & 7 & 6 & 7 & 6 & 6 & 7 & 6 & 6 & 7 & 6 & & \\
\hline year & .03 & .04 & .03 & .04 & .04 & .03 & .04 & .03 & .05 & .06 & & \\
\hline Locality & 22 & 22 & 11 & 21 & 44 & 11 & 44 & 18 & 33 & 18 & & \\
\hline Cultivated plant & $\mathrm{Sc}$ & $\mathrm{Sc}$ & $\mathrm{Sc}$ & $\mathrm{Sc}$ & $\mathrm{Sc}$ & $\mathrm{Sc}$ & $\mathrm{Sc}$ & $\mathrm{Sc}$ & $\mathrm{Sc}$ & $\mathrm{Sc}$ & & \\
\hline Percent ground cover by the cultivated plant & 50 & 70 & 60 & 70 & 60 & 70 & 55 & 90 & 60 & 90 & & \\
\hline Percent ground cover by weeds & 60 & 15 & 35 & 50 & 25 & 30 & 25 & 10 & 25 & 10 & & \\
\hline Soil unit & $\begin{array}{l}n \\
a \\
0\end{array}$ & 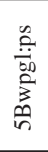 & 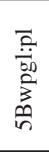 & 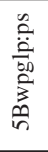 & $\begin{array}{l}\stackrel{n}{0} \\
\stackrel{n}{b 0} \\
\sum_{0}^{2} \\
\text { nै }\end{array}$ & $\begin{array}{l}n \\
0 \\
0 \\
0\end{array}$ & 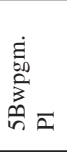 & 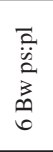 & $\begin{array}{l}n \\
2 \\
0 \\
0\end{array}$ & 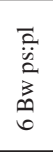 & & \\
\hline Number of species in the releve & 20 & 16 & 16 & 17 & 16 & 23 & 15 & 21 & 22 & 20 & & 19 \\
\hline \multicolumn{13}{|l|}{ I. Ch. D. Arnoserido-Scleranthetum } \\
\hline Arnoseris minima & 3 & + & 1 & 2 & + & 1 & 1 & 1 & + & 1 & $\mathrm{~V}$ & 830 \\
\hline Teesdalea nudicaulis & 1 & 1 & 2 & 1 & 2 & 2 & & & 1 & & IV & 725 \\
\hline \multicolumn{13}{|l|}{ II. Ch.D. Arnoseridenion minimae } \\
\hline Veronica dillenii & 1 & + & & & + & + & & + & + & + & IV & 110 \\
\hline Anthoxanthum aristatum & & & & + & & + & & + & & + & II & 40 \\
\hline Scleranthus annuиs & & & & & & + & & + & & + & II & 30 \\
\hline Spergula morisonii & 1 & & + & & + & & & + & & & II & 80 \\
\hline \multicolumn{13}{|l|}{ III. Ch. D.Papaveretum argemones } \\
\hline Arabidopsis thaliana & + & + & + & + & + & + & 1 & 1 & 1 & + & $\mathrm{V}$ & 220 \\
\hline Veronica triphyllos & + & 1 & 1 & 1 & + & + & 1 & 1 & + & + & $\mathrm{V}$ & 300 \\
\hline Papaver argemone & 1 & & + & + & & + & & 1 & + & + & IV & 160 \\
\hline \multicolumn{13}{|l|}{ IV. Ch. Aperion spicae-venti } \\
\hline \multicolumn{13}{|l|}{ Centauretalia cyani } \\
\hline Centaurea cyanus & + & + & & + & & + & & + & & + & III & 60 \\
\hline Apera spica-venti & & & & + & & & + & & 1 & & II & 70 \\
\hline Anthemis arvensis & & & + & & + & + & + & & & & II & 40 \\
\hline Vicia angustifolia & & & & & + & & + & & + & & II & 30 \\
\hline \multicolumn{13}{|l|}{ V. Ch. Stellarietea mediae } \\
\hline Fallopia convolvulus & + & & + & + & + & + & & & & & III & 50 \\
\hline Myosotis arvensis & & & + & & + & + & + & & + & & III & 50 \\
\hline Viola arvensis & & & & + & & + & + & & 1 & & II & 80 \\
\hline Spergula arvensis & + & & & & + & & & + & & + & II & 40 \\
\hline Polygonum aviculare & & + & & & & + & & + & & + & II & 40 \\
\hline Raphanus raphanistrum & & + & & & & & & + & & + & II & 30 \\
\hline Polygonum lapathifolium subsp. pallidum & & & & & & & & + & + & + & II & 30 \\
\hline \multicolumn{13}{|l|}{ VI. Accompanying species } \\
\hline Rumex acetosella & + & & + & + & + & + & + & & + & & IV & 70 \\
\hline Myosotis stricta & + & & & 1 & + & + & + & & + & & III & 100 \\
\hline Veronica arvensis & + & + & + & & & + & 1 & & + & & III & 100 \\
\hline Erophila verna & + & + & & + & & & 1 & & + & & III & 90 \\
\hline Taraxacum officinale & + & + & & & & + & & + & & + & III & 50 \\
\hline Cirsium arvense & + & + & + & & & & & & & & II & 30 \\
\hline Trifolium repens & & & + & & + & & & & + & & II & 30 \\
\hline Equisetum arvense & + & & & & & + & & + & & + & II & 30 \\
\hline Galeopsis tetrahit & & & & & & & & + & 1 & + & II & 70 \\
\hline
\end{tabular}

Sporadic species: III - Papaver dubium 8(+), 10(+); IV - Anchusa arvensis 6(r); Rhinanthus serotinus 2(+); Vicia villosa 7(+); V - Conyza canadensis 8(+), 10(+); Stellaria media 4(+), 9(+); Chenopodium album 4(+), 9(+); Capsella bursa-pastoris $8(+), 10(+)$; Geranium pusillum 5(+); VI - Erodium cicutarium 1(+), 6(+); Achillea millefolium 1(+), 4(+); Cerastium holosteoides 3(+), 5(+); Myosurus minimus 3(+), 7(+); Equisetum sylvaticum (+); Luzula arvensis $9(+) ;$ Galium aparine $9(+) ;$ Cardaminopsis arenosa 9(+); Cerinthe minor 2(+); Ranunculus sardous 3(+); Agrostis stolonifera 4(+); Sedum maximum 9(+); Knautia arvensis 8(+), 10(+); Veronica verna $8(+), 10(+)$; Convolvulus arvensis $6(+)$; Allium vineale $6(+)$; Elymus repens $1(+)$; Comments: numbers after species inform about the releve in the table.

$\mathrm{S}$ - phytosociological constancy, D - coverage index. 
Table 3

Papaveretum argemones (Libb. 1932) Krusem. Et Vlieg. 1939.

\begin{tabular}{|c|c|c|c|c|c|c|c|c|c|c|c|c|}
\hline Releve no. in the table & 1 & 2 & 3 & 4 & 5 & 6 & 7 & 8 & 9 & 10 & \multirow{9}{*}{\multicolumn{2}{|c|}{ 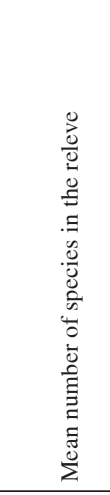 }} \\
\hline Releve no. in nature & 104 & 97 & 98 & 82 & 33 & 13 & 110 & 103 & 83 & 34 & & \\
\hline Date: month & 7 & 7 & 7 & 6 & 6 & 7 & 6 & 6 & 7 & 7 & & \\
\hline year & .05 & .05 & .03 & .04 & .04 & .03 & .04 & .03 & .03 & .06 & & \\
\hline Locality & 39 & 33 & 33 & 29 & 41 & 25 & 42 & 39 & 29 & 16 & & \\
\hline Cultivated plant & $\mathrm{Sc}$ & $\mathrm{Sc}$ & $\mathrm{Sc}$ & $\mathrm{Sc}$ & Sc & Miesz.j & $\mathrm{Sc}$ & $\mathrm{Sc}$ & $\mathrm{Sc}$ & $\mathrm{Sc}$ & & \\
\hline $\begin{array}{l}\text { Percent ground cover by the cultivated } \\
\text { plant }\end{array}$ & 80 & 60 & 75 & 60 & 60 & 75 & 60 & 80 & 60 & 60 & & \\
\hline Percent ground cover by weeds & 35 & 45 & 45 & 10 & 50 & 5 & 15 & 35 & 10 & 50 & & \\
\hline Soil unit & $\begin{array}{l}\bar{p} \\
\dot{0} \\
0 \\
z \\
0 \\
0\end{array}$ & 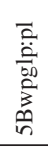 & 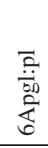 & 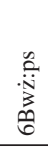 & 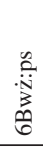 & $\begin{array}{l}\overline{00} \\
\dot{00} \\
0 \\
0 \\
0 \\
n \\
n\end{array}$ & 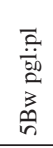 & $\begin{array}{l}\overrightarrow{0} \\
\text { तु. }\end{array}$ & $\begin{array}{l}\vec{a} \\
\text { : } \\
\text { : }\end{array}$ & 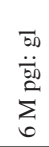 & & \\
\hline \multirow[t]{2}{*}{ Number of species in the releve } & 16 & 20 & 25 & 27 & 23 & 22 & 17 & 17 & 30 & 24 & \multicolumn{2}{|c|}{22} \\
\hline & & & & & & & & & & & $\mathrm{S}$ & $\mathrm{D}$ \\
\hline \multicolumn{13}{|l|}{ I. Ch. Papaveretum argemones } \\
\hline Papaver argemone & 2 & 2 & 1 & 1 & 1 & 1 & 1 & 2 & 2 & 1 & $\mathrm{~V}$ & 1000 \\
\hline Arabidopsis thaliana & 1 & + & 1 & & + & + & & 1 & 1 & 1 & IV & 280 \\
\hline Veronica triphyllos & & + & 1 & & + & + & 1 & & + & 1 & IV & 190 \\
\hline \multicolumn{13}{|l|}{ II. Ch. Aperion spicae-venti } \\
\hline \multicolumn{13}{|l|}{ Centauretalia cyanii: } \\
\hline Apera spica-venti & + & 1 & + & + & + & + & + & + & + & + & $\mathrm{V}$ & 140 \\
\hline Anthemis arvensis & + & + & + & + & + & & + & + & + & 1 & IV & 130 \\
\hline Centaurea cyanus & + & 1 & + & & 1.1 & & + & + & & 1 & IV & 190 \\
\hline Lithospermum arvense & + & & 1 & & 3 & & & + & & 2 & III & 620 \\
\hline Vicia hirsuta & + & & + & + & & & & + & + & & III & 50 \\
\hline Vicia tetrasperma & & & & & + & + & & & & + & III & 30 \\
\hline Veronica dillenii & & & + & & + & & & & & + & II & 30 \\
\hline Vicia villosa & & & & & + & & + & & & + & II & 30 \\
\hline Vicia angustifolia & & & + & + & & & & & + & & II & 30 \\
\hline Rumex acetosella & & & + & + & & & & & + & & II & 30 \\
\hline \multicolumn{13}{|l|}{ III. Ch. Stellarietea mediae } \\
\hline Myosotis arvensis & + & + & + & + & + & + & + & + & + & + & $\mathrm{V}$ & 100 \\
\hline Viola arvensis & + & 1 & + & & + & + & & + & & + & IV & 110 \\
\hline Geranium pusillum & + & & + & & + & & + & + & & + & III & 60 \\
\hline Capsella bursa-pastoris & & + & & + & & & + & & + & & II & 40 \\
\hline Fallopia convolvulus & & + & & & + & & + & & & + & II & 40 \\
\hline$\underline{\text { Spergula arvensis }}$ & & & + & + & & + & & & + & & II & 40 \\
\hline Conyza canadensis & & + & & + & & + & & & + & & II & 40 \\
\hline Matricaria maritima subsp. inodora & & & & & + & + & & & & + & II & 30 \\
\hline Chenopodium album & & & + & + & & & & & + & & II & 30 \\
\hline \multicolumn{13}{|l|}{ IV. Accompanying species } \\
\hline Arenaria serpyllifolia & 1 & + & 1 & & + & + & + & 1 & 2 & 1 & $\mathrm{~V}$ & 415 \\
\hline Myosotis stricta & + & + & + & 1 & + & + & + & + & 1 & 1 & $\mathrm{~V}$ & 220 \\
\hline Erophila verna & & + & 1 & + & + & & + & 1 & + & + & IV & 160 \\
\hline Veronica arvensis & + & 1 & & + & & + & & + & + & + & IV & 110 \\
\hline Equisetum arvense & & + & + & + & + & & & & + & + & III & 60 \\
\hline Galium aparine & & & & + & + & & + & & + & + & III & 50 \\
\hline Trifolium repens & & & & + & & + & & & + & & II & 30 \\
\hline Melandrium album & & & & + & & + & & & + & & II & 30 \\
\hline Erodium cicutarium & & & & + & & + & & & + & & II & 30 \\
\hline Allium vineale & & & + & & + & & & & & + & II & 30 \\
\hline Rhinanthus serotinus & & + & & + & & & & & + & & II & 30 \\
\hline
\end{tabular}

Sporadic species: II - Agrostemma githago 3(+), 6(+); Scleranthus annuus 2(+), 3(+); Arnoseris minima 6(+); III - Sonchus arvensis 4(+), 9(+); Sinapis arvensis 4(+), $9(+)$; Melandrium noctiflorum 4(+), 9(+); Sinapis arvensis 4(+), 9(+); Papaver rhoeas 4(+), 9(+); Lamium amplexicaule 6(+), 7(+); Stellaria media $6(+) ;$ Rumex crispus 7(+); IV - Achillea millefolium 5(+), 10(+); Sedum acre 5(+), 10(+); Artemisia arvensis 4(+), 9(+); Daucus carota 4(+), 9(+); Vicia cracca 4(+), 9(+); Knautia arvensis $1(+), 8(+)$; Hypericum perforatum 1(+), 8(+); Pimpinella saxifraga 1(+), 8(+); Tanacetum vulgare 6(+); Potentilla argentea 7(+); Taraxacum officinale 6(+); Senecio vernalis 3(+); Hieracium pilosella 3(+); Trifolium dubium 2(+); Trifolium medium 2(+);

Comments: numbers after species inform about the releve in the table.

$\mathrm{S}$ - phytosociological constancy, D - coverage index. Sc-Secale cereale 


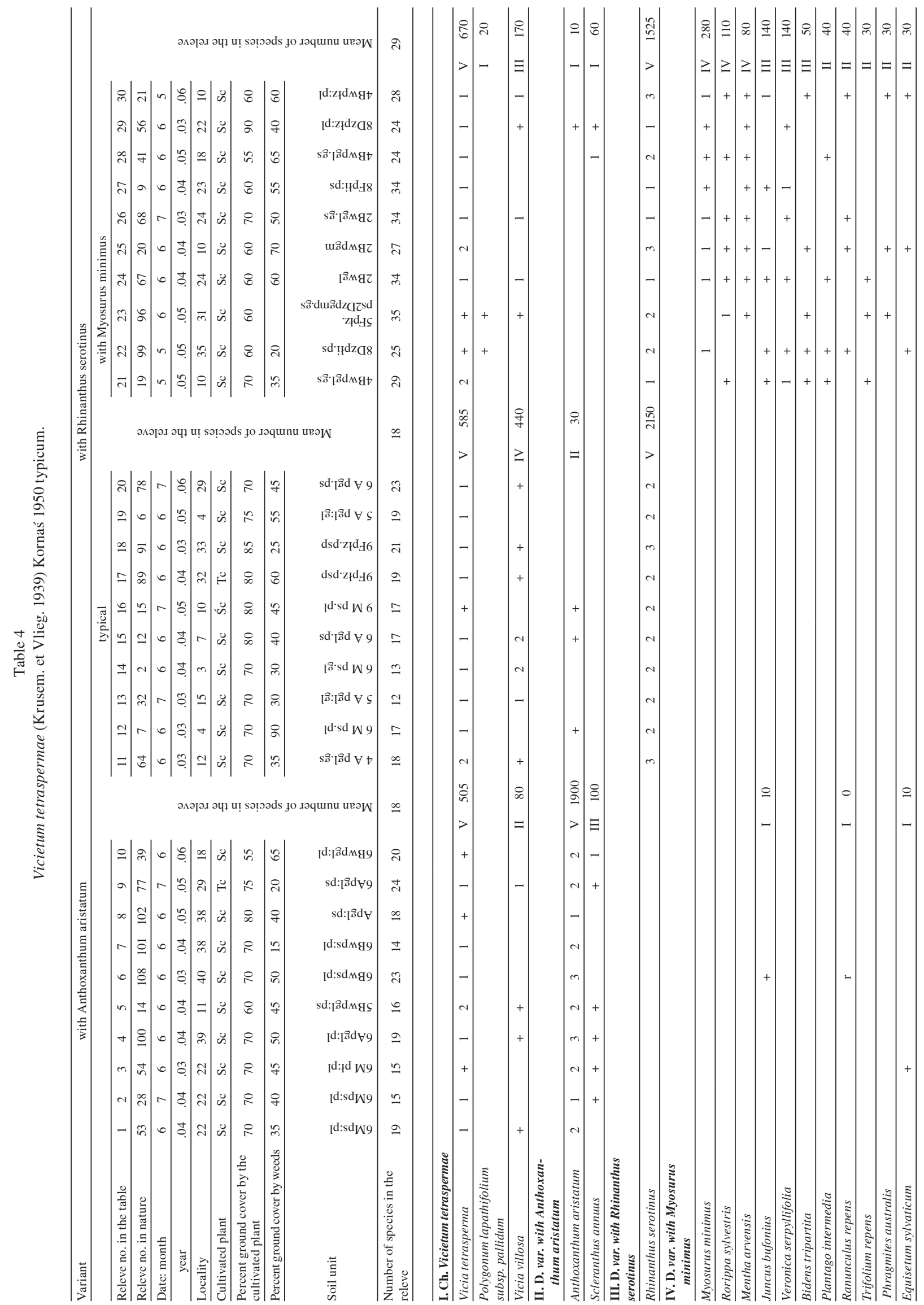




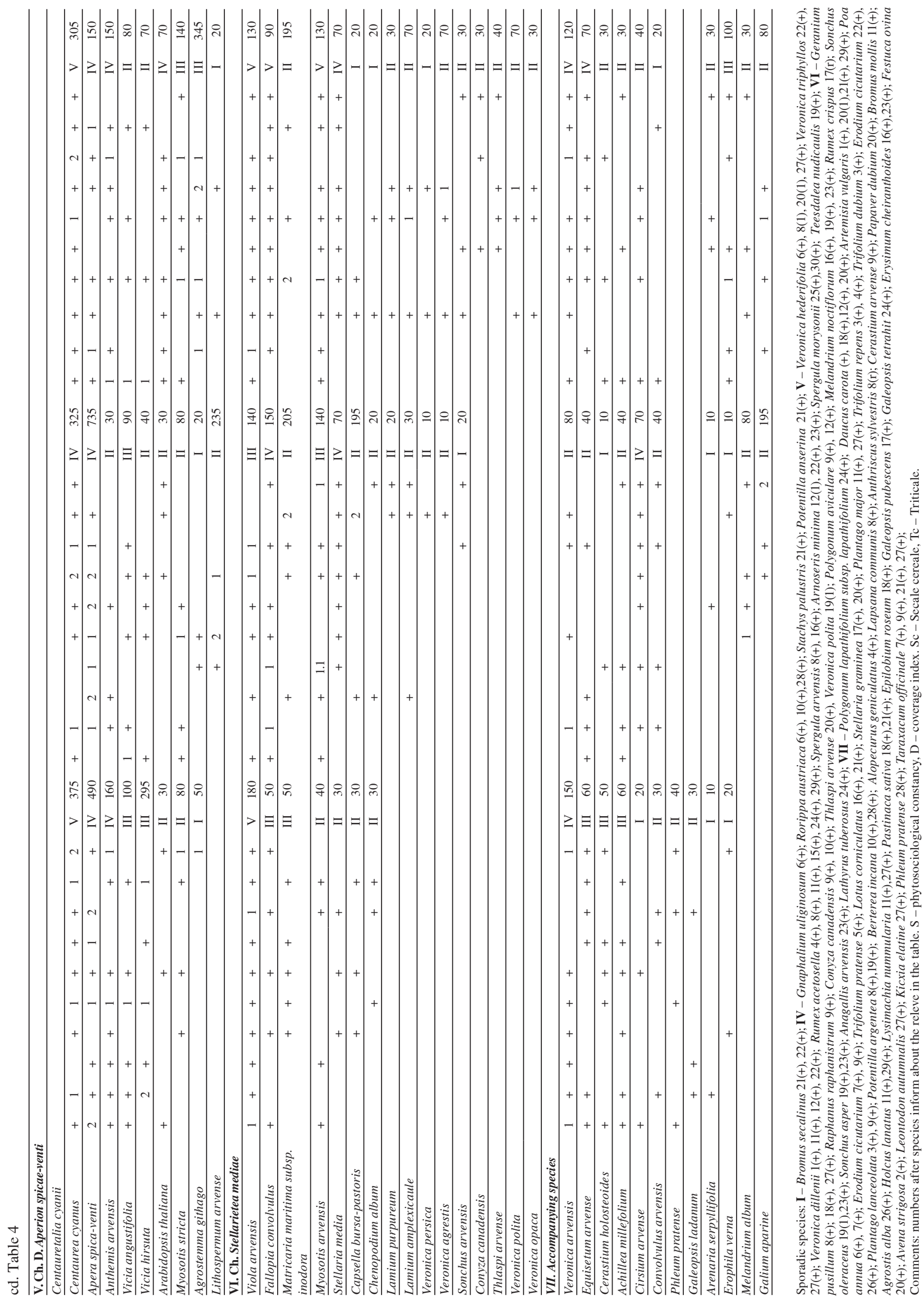




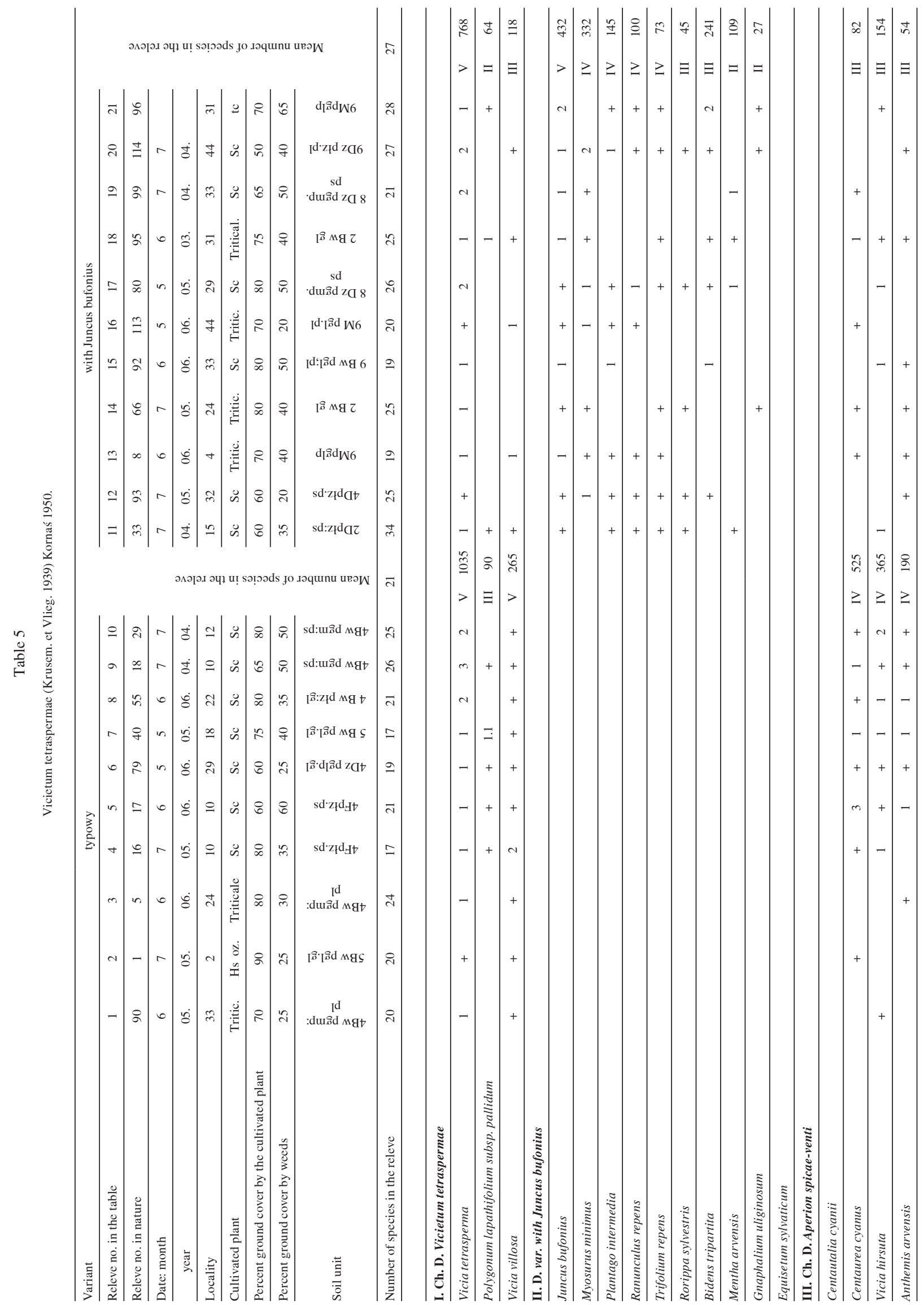




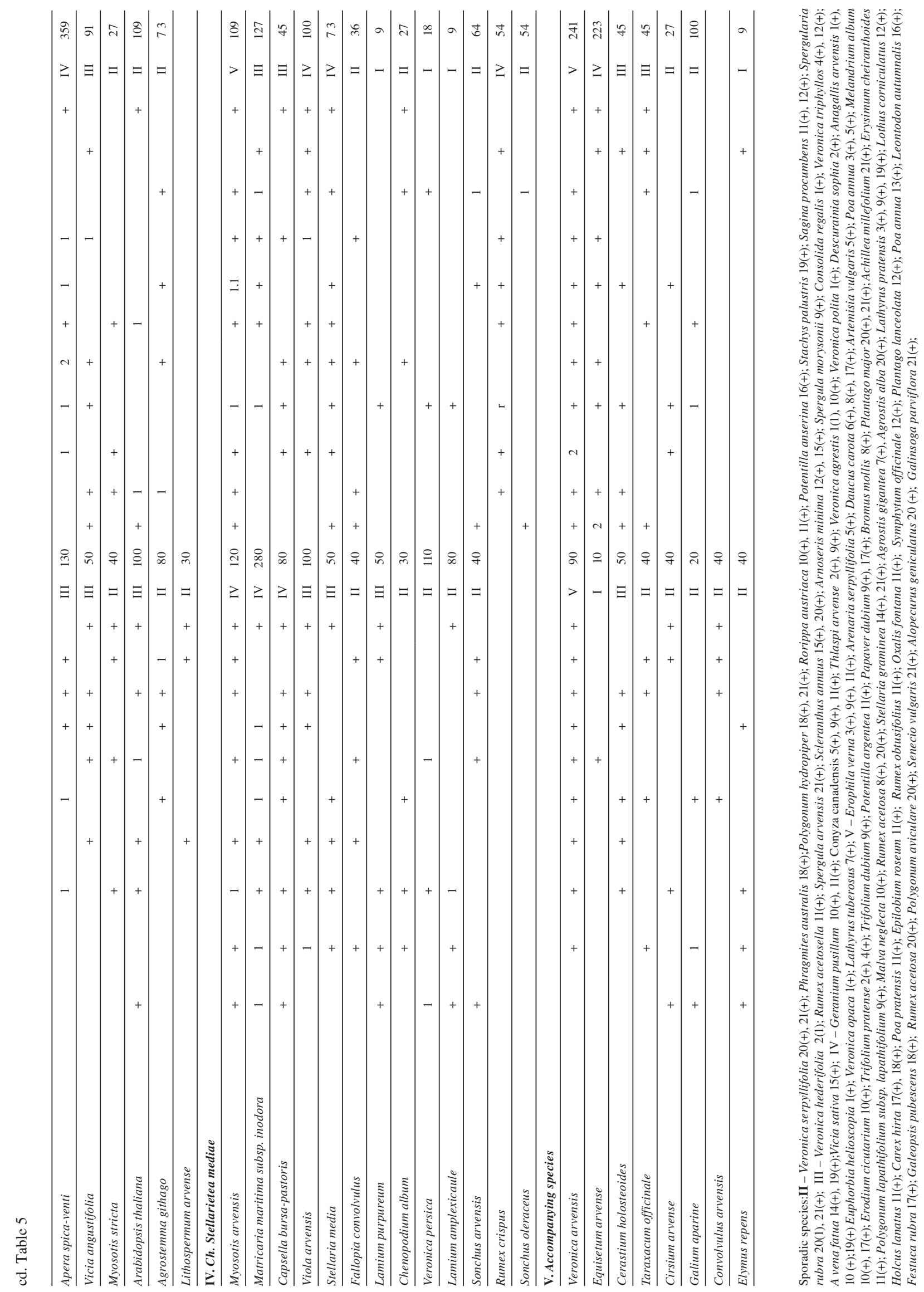


Table 6

Aphano-Matricarietum R.Tx. 1937.

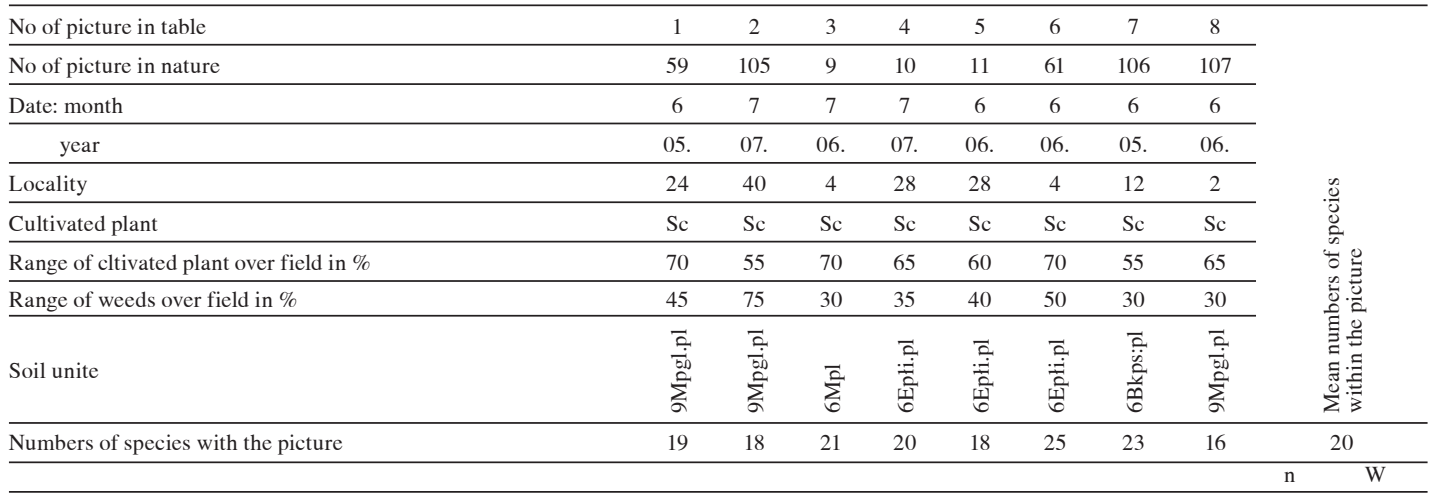

I.CH.D. Aphano-Matricarietum

Aphanes arvensis

Veronica hederifolia

Matricaria maritima subsp. inodora

II. Ch. Aperion spicae-venti

\begin{tabular}{|c|c|c|c|c|c|c|c|c|c|c|}
\hline $\mathrm{Cel}$ & & & & & & & & & & \\
\hline Anthemis arvensis & + & + & + & + & + & + & & + & 7 & 87 \\
\hline Apera spica-venti & & 2 & & 1 & 1 & 1 & 1 & + & 6 & 481 \\
\hline Scleranthus annuиs & 1 & + & & + & & + & + & & 5 & 112 \\
\hline Teesdalea nudicaulis & 1 & & 1 & & + & & + & & 4 & 150 \\
\hline Arnoseris minima & + & + & & & & + & & $\mathrm{r}$ & 4 & 37 \\
\hline Lithospermum arvense & & & & 1 & + & + & 1 & & 4 & 150 \\
\hline Arabidopsis thaliana & & & 1 & & & + & + & & 3 & 87 \\
\hline Vicia angustifolia & + & & & & + & & + & & 3 & 37 \\
\hline Vicia villosa & + & & + & & & + & & & 3 & 37 \\
\hline Veronica dillenii & & & 1 & & & 1 & + & & 3 & 137 \\
\hline Centaurea cyanus & + & & & & & + & & + & 3 & 37 \\
\hline \multicolumn{11}{|l|}{ III. Stellarietea mediae } \\
\hline Viola arvensis & + & + & + & + & + & + & + & + & 8 & 100 \\
\hline Geranium pusillum & & & + & + & & + & + & & 4 & 50 \\
\hline Spergula arvensis & & & + & + & + & & & + & 4 & 50 \\
\hline Fallopia convolvulus & & & & + & & + & + & + & 4 & 50 \\
\hline Conyza canadensis & & & & & + & & + & & 2 & 25 \\
\hline \multicolumn{11}{|l|}{ IV. Accompanyig species } \\
\hline Veronica arvensis & & & + & + & & + & + & + & 5 & 62 \\
\hline Juncus bufonius & + & 1 & + & & & + & & & 4 & 100 \\
\hline Galium aparine & + & & + & + & & + & & & 4 & 50 \\
\hline$\overline{\text { Anthoxanthum aristatum }}$ & & 2 & & + & & & + & + & 4 & 256 \\
\hline Pоа аппиа & & & + & + & & + & & + & 4 & 40 \\
\hline Galeopsis ladanum & & + & & + & & & + & & 3 & 37 \\
\hline Rhinanthus serotinus & 2 & & 1 & & & & + & & 3 & 287 \\
\hline Cerastium holosteoides & & + & + & & + & & & & 3 & 37 \\
\hline Rumex acetosella & & & & + & & + & & + & 3 & 37 \\
\hline Leontodon autumnalis & + & & & + & & & + & & 3 & 37 \\
\hline$\overline{\text { Hypochoeris glabra }}$ & & + & + & & & & + & & 3 & 37 \\
\hline Holcus lanatus & & & & + & & + & + & & 3 & 37 \\
\hline Polygonum hydropiper & & & + & & + & & & & 2 & 25 \\
\hline Elymus repens & & + & & & & + & & & 2 & 25 \\
\hline Ranunculus repens & & + & + & & & & & & 2 & 25 \\
\hline$\overline{\text { Bidens tripartita }}$ & + & 1 & & & & & & & 2 & 75 \\
\hline Polygonum hydropiper & 1 & 1 & & & & & & & 2 & 125 \\
\hline Carex hirta & + & & & & & + & & & 2 & 25 \\
\hline Vicia tetrasperma & + & + & & & & & & & 2 & 25 \\
\hline$\overline{\text { Veronica verna }}$ & + & & & & & & + & & 2 & 25 \\
\hline Plantago lanceolata & + & & & & & + & & & 2 & 25 \\
\hline Ranunculus sardous & & & & + & & & & + & 2 & 25 \\
\hline Convolvulus arvensis & & & & & + & & & + & 2 & 25 \\
\hline
\end{tabular}

Sporadic species: III - Capsella bursa-pastoris 5(+); IV - Plantago intermedia 3(+); Luzula arvensis 1(+); Trifolium dubium 3(+); Tanacetum vulgare 3(+); Equisetum arvense 7(+); Spergularia rubra 2(+); Lisymachia vulgaris 2(+); Potentilla anserina 5(+); Taraxacum officinale 5(+); Rorippa sylvestris 5(+); Polygonum aviculare 7(+); Cirsium arvense 8(+); Erodium cicutarium 8(+); Vicia hirsuta 6(+);

Comments: numbers after species inform about the releve in the table. S - phytosociological constancy, D - coverage index. 
Table 7

Community with species characteristic for Polygono-Chenopodion.

\begin{tabular}{|c|c|c|c|c|c|c|c|c|c|c|c|}
\hline Releve no. in the table & 1 & 2 & 3 & 4 & 5 & 6 & 7 & 8 & 9 & 10 & \multirow{9}{*}{ 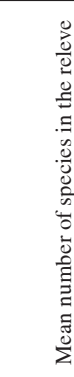 } \\
\hline Releve no. in nature & 109 & 30 & 22 & 71 & 31 & 81 & 42 & 115 & 70 & 116 & \\
\hline Date: month & 7 & 7 & 6 & 7 & 6 & 5 & 5 & 6 & 7 & 7 & \\
\hline year & 04. & 05. & 06. & 05. & 06. & 06. & 05. & 03. & 04. & 04. & \\
\hline Locality & 38 & 12 & 10 & 24 & 12 & 29 & 18 & 44 & 24 & 39 & \\
\hline Cultivated plant & HsvAs & $\mathrm{Tc}$ & As & $\mathrm{Hs}$, As & $\mathrm{Hv}$ & $\mathrm{Tv}$ & As & $\mathrm{Sc}$ & $\mathrm{Sc}$ & $\mathrm{Hs}, \mathrm{As}$ & \\
\hline Percent ground cover by the cultivated plant & 90 & 95 & 80 & 70 & 80 & 70 & 65 & 60 & 90 & 85 & \\
\hline Percent ground cover by weeds & 20 & 30 & 20 & 55 & 25 & 20 & 55 & 30 & 35 & 20 & \\
\hline Soil unit & 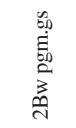 & 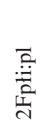 & 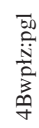 & 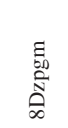 & $\begin{array}{l}\text { कD } \\
\text { N } \\
\overline{2} \\
\text { त } \\
\text { N }\end{array}$ & $\begin{array}{l}\text { कD } \\
\text { 命 } \\
\text { N }\end{array}$ & 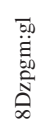 & 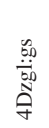 & $\begin{array}{l}\overline{50} \\
\overline{0.0} \\
\overrightarrow{0} \\
\hat{F}\end{array}$ & 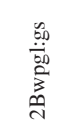 & \\
\hline Number of species in the releve & 18 & 29 & 17 & 28 & 22 & 31 & 25 & 24 & 25 & 21 & 24 \\
\hline
\end{tabular}

\begin{tabular}{c}
\hline I. Ch. Polygono-Chenopodion \\
Polygono-Chenopodietalia
\end{tabular}

\begin{tabular}{|c|c|c|c|c|c|c|c|c|c|c|c|c|}
\hline Polygono-Chenopodietalia & & & & & & & & & & & & \\
\hline Veronica persica & 1 & 1 & & 2 & & 1 & 2 & + & + & 1 & IV & 570 \\
\hline$\underline{\text { Stellaria media }}$ & 1 & 1 & + & & & 1 & & 1 & + & + & IV & 230 \\
\hline Chenopodium album & + & + & 1 & 1 & 1 & + & & & + & + & IV & 200 \\
\hline Lamium purpureum & + & + & + & + & 1 & + & & & & + & IV & 110 \\
\hline Matricaria maritima subsp. inodora & + & + & + & 1 & + & + & & & + & + & IV & 120 \\
\hline Lamium amplexicaule & + & + & & & & + & + & + & + & + & IV & 70 \\
\hline Veronica polita & + & 2 & & + & & & 1 & & & + & III & 255 \\
\hline Thlaspi arvense & 1 & + & & 1 & & & & + & + & + & III & 140 \\
\hline Veronica agrestis & & 1 & & 1 & + & & + & & & + & III & 130 \\
\hline Anagallis arvensis & + & & & + & & & & + & 1 & + & III & 90 \\
\hline Euphorbia helioscopia & & + & & & + & + & + & & & 1 & III & 90 \\
\hline Chenopodium polyspermum & + & & & + & + & & + & & & + & III & 50 \\
\hline Sinapis arvensis & + & & & + & & + & + & & & + & III & 50 \\
\hline Sonchus oleraceus & & & & + & & + & + & & & & II & 30 \\
\hline Sonchus asper & & + & & & + & & & + & & & II & 30 \\
\hline
\end{tabular}

II. Ch. Aperion spicae-venti

Centauretalia cyanii

\begin{tabular}{|c|c|c|c|c|c|c|c|c|c|c|c|c|}
\hline Vicia angustifolia & + & + & + & & + & + & & + & & + & IV & 70 \\
\hline Centaurea cyanus & & & + & & + & & + & + & + & & III & 50 \\
\hline Polygonum lapathifolium subsp. pallidum & & & & + & + & + & + & & & & II & 40 \\
\hline Anthemis arvensis & & & & + & + & & & + & & & II & 30 \\
\hline Vicia sativa & & + & + & + & & & & & & & II & 30 \\
\hline \multicolumn{13}{|l|}{ III. Ch. Stellaria mediae } \\
\hline Viola arvensis & & + & + & & + & & + & 1 & 1 & & III & 140 \\
\hline Myosotis arvensis & + & + & + & + & & & & & + & + & III & 60 \\
\hline Capsella bursa-pastoris & & + & + & + & 1 & + & & & & & III & 90 \\
\hline Fallopia convolvulus & & & + & & 1 & + & + & & & & II & 80 \\
\hline Spergula arvensis & & + & + & + & & + & & & & & II & 40 \\
\hline Geranium pusillum & & + & & & + & & & + & & & II & 30 \\
\hline Raphanus raphanistrum & & & & + & & & + & + & & & II & 30 \\
\hline
\end{tabular}

Raphanus raphanistrum

IV. Accompanying species

\begin{tabular}{|c|c|c|c|c|c|c|c|c|c|c|c|c|}
\hline Galium aparine & 1 & & & 1 & & + & & & + & 1 & III & 170 \\
\hline Veronica arvensis & & + & 1 & 1 & + & + & & + & + & & IV & 150 \\
\hline Artemisia vulgaris & & + & & & & + & 1 & & & & II & 70 \\
\hline Polygonum lapathifolium subsp. lapathifolium & & + & 1 & & & + & & & & & II & 70 \\
\hline Taraxacum officinale & & & + & + & & + & & & + & & II & 40 \\
\hline Equisetum arvense & & & + & & + & & & + & + & & II & 40 \\
\hline Plantago intermedia & + & & & + & & + & & & & + & II & 40 \\
\hline Alopecurus geniculatus & + & & & + & & & & & & + & II & 30 \\
\hline
\end{tabular}

Sporadic species: I - Galinsoga parviflora 6(1), 10(+); Melandrium noctiflorum 5(+), 9(+); Rumex crispus 9(+); II - Apera spica-venti 7(+), 9(2); Arabidopsis thaliana 8(1), 9(+); Vicia hirsuta 2(1), 5(+); Veronica hederifolia 9(+); Vicia villosa 8(+): III - Polygonum aviculare 4(+), 6(+); Echinochloa crus-galli 7(+); Senecio vulgaris 6(+); Rumex acetosella 5(+); IV - Myosotis stricta 8 (1), 9(1); Plantago major 2(+), 6(+); Cirsium arvense 2(+), 6(+); Erodium cicutarium 5(+), 9(+); Stellaria graminea 7(+), 9(+); Trifolium repens 2(+), 6(+); Cerastium holosteoides 8(+), 9(+); Erophila verna 8(1), 9(+); Poa annua 1(+), 10(+); Elymus repens 6(+), 7(+); Galeopsis tetrahit 4(+), 6(+); Convolvulus arvensis 2(+), 5(+); Myosurus minimus 8(1), 9(+); Juncus bufonius 1(+), 10(+); Lappa minor 3(+); Polygonum persicaria 4(+); Matricaria suaveolens 4(+); Chenopodium hybridum 6(+); Hypochoeris glabra 7(+); Tanacetum vulgare 6(+), 7(+); Helichrysum arenarium 8(+); Senecio vernalis 8(+); Viola tricolor $8(+)$; Leontodon autumnalis $7(+) ;$ Polygonum hydropiper $7(2)$ Potentilla anserina 2(+); Rorippa sylvestris 2(+); Trifolium dubium 4(+); Galeopsis ladanum 7(+); Galeopsis pubescens 2(+); Rumex acetosa $7(+) ;$ Achillea millefolium 7(+);

Daucus carota 6(+); Festuca rubra 8(+); Comments: numbers after species inform about the releve in the table. 
Typical patches of Vicietum tetraspermae were rarely noted in the study area. They were distinguishable by higher cover of characteristic species and the lack of acidophilous, calcareous and higrophilous species. These phytocenoses developed on brown lixiviated soils, alluvial soils, and degraded black soils, classified as good and very good rye complexes. They were distinguishable by higher cover of Vicia hirsuta and Centaurea cyanus.

Patches with the participation of hygrophilous species were noted much more frequently. The plots of this variant were the floristically richest phytocenoes in cereal crops of the Park. They were built up of 88 species, and the mean number of species in one plot was on average 27 . The patches were distinguishable by the occurrence of a numerous group of hygrophilous species, of which the highest persistence and cover reached the following: Juncus bufonius, Myosurus minimus, Plantago intermedia, Ranunculus repens and Triforium repens. These phytocenoses, comparing with the typical ones, were also characterised by a larger share of Apera spica-venti.

On the poorest soils, classified as poor and good rye complexes, phytocenoses with the mass occurrence of Anthoxanthum aristatum (cover frequently over $30 \%$ ) were observed. These floristically poorest patches, considering the phytocenoses of Vicietum tetraspermae, were also distinguishable by the smallest cover of characteristic species.

The frequent occurrence of Rhinanthus serotinus in some patches of Vicietum tetraspermae resulted in different physiognomy of the community. That specific combination of species is presented as the variant with Rhinanthus serotinus. Typical phytocenoses of the variant with Rhinanthus serotinus were developing in habitats similar in trophy to those of the variant with Anthoxanthum aristatum. They were also floristically poor. Some plots, classified as the subvariant with Myosurus minimus, were characterised by the occurrence of hygrophilous species. These phytocenoses, contrary to those mentioned above, developed on rich soils, included in a very good rye complex, wheat good and cereal-grazing complexes, formed of clayey sands, dusts and loam. The analyzed plots were distinguishable by the occurrence of hygrophilous species, e.g.: Myosurus minimus, Juncus bufonius, Plantago intermedia, Mentha arvensis, Rorippa sylvestris. In some patches Agrostemma githago and Centaura cyanus dominated.

\section{Aphano-Matricarietum R. Tx. 1937}

In cereal crops of mid-forest clearings of the following villages: Kąty, Skorupy, Augustówka, Człekówka and Ocznia, phytocenoses with Aphanes arvensis were recorded. Matricaria maritima subsp. inodora, noted frequently in other communities, occurred in the analyzed patches with a little cover. The presence of Veronica hederifolia, a rare taxon in the phytocenoses of the Landscape Park, was also sporadically observed in them. No stand of Chamomilla recutita, reported as a characteristic species of AphanoMatricarietum (Matuszkiewicz, 2001), was recorded in the study area. Phytocenoses of AphanoMatricarietum were observed mainly in rye crops on sandy, boggy and silt-peat soils of poor rye and poor cereal-grazing complexes. They developed on poor and periodically excessively wetted soils with a large share of acidophilous: Scleranthus annuus, Rumex acetosella, Arnoseris minima, Teesdalea nudicaulis, Anthemis arvensis, and hygrophilous species: Juncus bufonius, Polygonum hydropiper, Bidens tripartita and others. Moreover, Rhinanthus serotinus, numerously noted in different phytocenoses, was also frequently recorded in that association.

\section{Communities with species characteristic for the Polygono-Chenopodion alliance}

In spring cereal crops, on the most fertile soils of the studied area, classified as a very good rye complex, good wheat complex and strong cereal-grazing complex, communities with a large share of species characteristic for the Polygono-Chenopodion alliance (Tab. 7) were noted. Species such as Veronica persica, Lamiom purpureum, Matricaria maritima subsp. inodora and Euphorbia helioscopia were most frequent. In the floristic composition of these patches, the occurrence of nitrophilous species characteristic for higher syntaxonomical units, e.g. Lamium amplexicaule, Stellaria media, Thlaspi arvensis, Anagallis arvensis and Veronica agrestis, as well as accompanying species: Galium aparine and Veronica arvensis, was observed.

\section{DISCUSSION}

Segetal vegetation undergoes constant, dynamic transformations which significantly affect the development of communities without diagnostic species of defined associations (A n i o ł - K w i a t k o w s k a , 1990; Hołdyński, 1991; Siciński, 2003; Skrajna and S k r z y c z yń s ka, 2006).

Syntaxonomically differentiated and speciesrich phytocenoses accompanying cereal crops in the area of Mazowiecki Landscape Park retained the characteristic combination of species.

The poorest habitats of the study area were occupied by the commonly noted phytocenoses of Arnoserido-Scleranthetum. That sub-Atlantic association reaches in Poland its north-eastern geographical range (Matuszkiewicz, 2001). Patches of Arnoserido-Scleranthetum observed in the area of 
the Park were similar in their floristic composition to phytocenoses reported from central and western Poland (A n i o ł- K w i a tk ow s k a 1990; S i c iński, 1986, 2003; Skrzyczyńska and Skrajna, 2004; W a r cholińs k a , 1995; Wój c i k, 1965), however, they were better developed and floristically richer than those occurring in the north-eastern part of the lowland Nizina Południowopodlaska (S k r z y c z yń s k a , 1994; S k r z y c z y ń s k a and R z y mow ska, 2005). Most frequently, similarly like in many regions of Poland, patches of Vicietum tetraspermae were noted. However, due to the domination of poor habitats and a large share of rye crops in the crop structure, patches of the variants characterised by the mass occurrence of Anthoxanthum aristatum, Rhinanthus serotninus and hygrophilous species were noted. Floristically similar patches of Vicietum tertaspermae were recorded in the Mazowsze region by W ó j c i k (1965), in the Wysoczyzna Kałuszyńska upland by S k r z y c z yńs k a and S krajna (2004) and in the Podlaski Przełom Bugu mesoregion by S krzy c z y ń s ka and R z y mow ska (2005).

The large fragmentation of arable areas, the close proximity of associations of similar habitat requirements and an increase in fertilization level (especially on light soils) resulted in the development of phytocenoses of intermediate character ( $\mathrm{S}$ i c i ń s k i, 2003). Phytocenoses characterized by the occurrence of characteristic species of both the associations: Arnoserido-Scleranthetum and Papaveretum argemones were rarely noted. Similar communities of intermediate character were reported from various regions of Poland by the following authors: W ój c i k (1965) from the Mazowsze region, Skrajna and Skrzyczyńska (2006) from the Wysoczyzna Kałuszyńska upland, Siciński (1986) from the Załęczański Landscape Park, Hołdyński and Korniak (1994), R a tus zniak and S obisz (1999) and so on. As a result of a small share of warm and calcareous habitats, the Papaveretum argemones association in agrocenoses of the Mazowiecki Landscape Park was observed seldom. The phytocenosis is widespread in southern and central Poland (A ni oł-Kwiatkowska, 1990; S i c iń s k i, 2003; W a r c h o li ń s k a ,1988; $\mathrm{W} \mathrm{nuk}$, 1976). The floristically poorest form of the association, which differed in the occurrence of only the summer vegetation aspect, was described in the Suwałki Lake District (W ó j c i k, 2000).

In rye crops, impoverished patches of AphanoMatricarietum - a substitute community to Vicietum tetraspermae, occurring commonly in cereal crops in north-western Poland, were noted very seldom ( $\mathrm{M} \mathrm{a}$ t u s z ki e w i c z, 2001).

In spring cereal crops, favourable temperature and light conditions affected the development of vegetation patches similar in species composition to communities of the order Polygono-Chenopodietalia, accompanying root cultivations ( $\mathrm{S}$ i c i ń s k i, 2003; Hołdyński , 1991; Skrajna and S k r z y c z y ń s k a, 2006). In the agrocenoses of the Mazowiecki Landscape Park, they were mainly observed on more fertile soils in the agricultural buffer zone of the Park.

\section{CONCLUSIONS}

1. Four plant communities, Arnoserido-Scleranthetum, Papaveretum argemones, Vicietum tetraspermae and Aphano-Matricarietum, divided into lower syntaxonomical units, were identified in cereal agrocenoses of the Mazowiecki Landscape Park.

2. The Arnoserido-Scleranthetum association was common in poor habitats, whereas the association Vicietum tetraspermae was widespread in more fertile ones.

3. Fully-developed plots of the association Papaveretum argemones and patches of the community of intermediate character Arnoserido-ScleranthetumPapaveretum argemones were rarely noted in the study area.

4. Isolated patches of Aphano-Matricarietum were noted in the south-eastern part of the Park.

\section{REFERENCES}

Aniol-Kwiatkowska J., 1990: Zbiorowiska segetalne Wału Trzebnickiego. Florystyczno-ekologiczne studium porównawcze. / Segetal communities of Wał Trzebnicki. Floristic and ecological comparative study. Wyd. Uniw. Wr. Pr. Bot.: 46-230.

Hołdyński Cz., 1991: Flora segetalna, zróżnicowanie florystyczno-ekologiczne i przemiany szaty roślinnej pól uprawnych w aktualnych warunkach agroekologicznych Żuław Wiślanych. / Segetal flora, floristic and ecological diversity and transformations of the plant cover of cultivated fields in current agro-ecological conditions of the Vistula Delta Plain. Acta Acad. Agricult. Tech. Olst. Agricultura, 51 (403): 1-51.

Hołdyński Cz., Korniak T., 1994: Charakterystyka flory i zbiorowiska segetalnych Parku Krajobrazowego Pojezierze Iławskie. / Characteristics of flora and segetal communities of the Pojezierze Ilawskie Landscape Park. Acta Agrobot. 47 (1): 67-81.

Matuszkiewicz W., 2001: Przewodnik do oznaczania zbiorowisk roślinnych Polski. Państwowe Wydawnictwo Naukowe, Warszawa.

Rat uszniak I., S obisz Z., 1999: Zbiorowiska segetalne Parku Krajobrazowego „Dolina Słupi”. Część I. / Segetal communities of the „Stupia River Valley” Landscape Park. Part I. Bad. Fizjograf. Nad Polską Zach. Ser. B - Botanica: 4897-110. 
Siciński J., 1986: Agrofitocenozy Załęczańskiego Parku Krajobrazowego. / Agrophytocenoses of the Załęczański Landscape Park. Acta Univ. Lodz., Folia sozol. 2: 523-566.

Siciński J. T., 2003: Agrofitocenozy dorzecza środkowej Warty i Bzury - stan, dynamika i zagrożenia. / Agrophytocenoses of the middle Warta and Bzura rivers basin - the state, dynamics and threats. Wyd. Uniwersytetu Łódzkiego. Rozp.: 1-69.

Skrajna T., Skrzyczyńska., 2006: Zbiorowiska przejściowe i kadłubowe występujące w łanach zbóż Wysoczyzny Kałuszyńskiej. / Temporary and impoverished communities occurring in corn-fields of the Kałuszyńska Upland. / Acta Sci. Pol., Biologia, 5 (1-2): 59-72.

Skrzyczyńska J., Skrajna T., 2004: Zbiorowiska polne Wysoczyzny Kałuszyńskiej. Cz. 1. Zespoły zbożowe. / Field comunities of the Kałuszyńska Upland. Part 1. Cereal communities.

Skrzyczyńska J., 1994: Studia nad florą i zbiorowiskami segetalnymi Wysoczyzny Siedleckiej. / Studies on the flora and segetal communities of the Siedlce Upland. Wyd. WSR-P w Siedlcach. Rozp. 39: 5-145.

Skrzyczyńska J., Rzymowska Z., 2005: Zbiorowiska roślinne pól uprawnych Podlaskiego Przełomu Bugu. Cz. I. Zbiorowiska zbożowe. / Plant communities of cultivated fields of Podlaski Przełom Bugu. Part 1. Communities of cereal crops.Acta Agrobot. 58 (1): 255-290.

Wa rcholińsk a U. A., 1988: Roślinność segetalna terenów rolniczych Puszczy Bolimowskiej i jej współczesne przemiany. / Segetal vegetation of agricultural areas of the Bolimów Primeval Forest and its contemporary transformations. Acta Agrobot. 58 (1): 255-290.

Warcholińska U. A.,1995: Arnoserido-Scleranthetum annui (Chouard 1925) R. Tx. 1937 corr. Matuszkiewicz Em. Warcholińska 1990 in Poland. Thaiszia - J. Bot. 5: 81-96.

Wnuk Z., 1976: Zbiorowiska chwastów segetalnych Pasma Przedborsko-Małogoskiego i przyległych terenów. Cz. I. Zbiorowiska upraw okopowych. / Segetal communities of the Przedborsko-Małagowskie Hills Range and adjacent areas. Part 1. Root crop communities. / Acta Univ. Lodz., Zesz. Nauk. UŁ, ser. II, 14: 123-177.
Wój ci k Z., 1965: Les Associations des chaps cultives en Masonie. I-ere partie: Les Associations messicoles. Ekol Pol. ser. A 13 (30): 1-42 (642-682).

Wójcik Z., 2000: Zbiorowiska Segetalne Pojezierza Suwalskiego. / Segetal communities of the Suwałki Lake District. Fragm.. Flor Geobot. ser. Polonica, 7: 167208.

\section{Zbiorowiska segetalne zbóż Mazowieckiego Parku Krajobrazowego}

\section{Streszczenie}

$\mathrm{W}$ pracy przedstawiono charakterystykę zbiorowisk wykształcających się w zasiewach zbóż na terenie Mazowieckiego Parku Krajobrazowego. Tradycyjne metody uprawy, duża mozaika pól, łąk i lasów oraz specyficzny mikroklimat, sprzyjają wykształcaniu się bogatych florystycznie i zróżnicowanych syntaksonomicznie agrofitocenoz. Na terenie Parku stwierdzono występowanie 4 zespołów (Arnoserido-Scleranthetum, Vicietum tetraspermae, Papaveretum argemones, Aphano-Matricarietum) i 2 zbiorowisk (ArnoseridoScleranthetum i Papaveretum argemones, z dominacją gatunków charakterystycznych rzędu PolygonoChenopodietalia). Najbardziej rozpowszechnionym i zróżnicowanym wewnętrznie był na glebach najsłabszych Arnoserido-Scleranthetum, a na zasobniejszych troficznie zespół Vicietum tetraspermae. Wymienione asocjacje częściej wykształcały się w oziminach sporadycznie w łanach zbóż jarych. Rzadko na badanym terenie spotykano płaty Papaveretum argemones oraz zbiorowiska o charakterze pośrednim. Płaty z dominacją gatunków charakterystycznych dla rzędu Polygono-Chenopodietalia notowano jedynie w lanach zbóż jarych głównie w otulinie Parku. W południowowschodniej części Parku występowały pojedyncze płaty Aphano-Matricarietum. 